\title{
Perceptual learning in visual category acquisition
}

\author{
HOWARD S. HOCK, ELIZABETH WEBB, and L. CLAYTON CAVEDO \\ Florida Atlantic University, Boca Raton, Florida
}

\begin{abstract}
The attribute structure of a set of dot patterns was studied by having subjects segment (parse) the dots of each pattern into parts or subunits by drawing circles around groups of dots from each pattern. These parsing data were obtained for subjects who had no prior experience with the patterns and for subjects who had previously learned to identify the patterns as members of one of four categories. Analyses of the parsing data indicated that category learning increased the salience of large subunits that were similar in orientation for patterns that were members of the same category. This evidence for perceptual learning was obtained even when the category training procedure required learning to identify the patterns individually, suggesting that attribute abstraction and item learning are not incompatible. It was also obtained without an increase in overall intersubject agreement. The latter result led us to question the usefulness of intersubject agreement as an index of category knowledge.
\end{abstract}

One of the strategies available to subjects learning the category membership of a set of stimuli is to determine the attributes that are likely to be shared by members of the same category but that are unlikely to be shared by members of contrasting categories (i.e., diagnostic attributes). Brooks (1978) refers to this strategy as analytic. The relative difficulty of analytic category learning depends on how readily shared/discriminative attributes can be abstracted (i.e., tested for diagnosticity independent of other attributes).

We would expect most subjects to begin category training analytically by testing attributes that are precategorically salient (a similar assumption is made in Fried and Holyoak's, 1984, category density model). These are the attributes that subjects would be most likely to notice and remember in examining the stimuli individually. If the precategorically salient attributes are not diagnostic of category membership, the subject could persist in an analytic strategy, continuing the search for attributes that are likely to be shared by members of the same category, but not by members of contrasting categories. This would lead the subject to consider, as potentially diagnostic of category membership, attributes that were not the most salient prior to category training. Successful category learning based on searching for shared/discriminative attributes could therefore be accompanied by increases, relative to precategorical values, in the salience of attributes shared by members of the same category. (An alterna-

The research reported in this paper was supported by Grants DAHC1978-G-002 and MDA903-82-C-0317 from the Army Research Institute. Elizabeth Webb is currently at Notre Dame College. Clay Cavedo is currently at Pennsylvania State University. We thank Pamela Hart, Linda Field, Cathy Stutin, and Alexandra Bates for their help in analyzing the data, and Edward O'Brien for his careful reading of the manuscript. Please address correspondence to Howard Hock, Department of Psychology, Florida Atlantic University, Boca Raton, FL 33431. tive strategy could result in category learning without changes in attribute salience; Brooks, 1978, and Medin, Dewey, and Murphy, 1983, demonstrated that under certain conditions, subjects forgo testing for diagnostic attributes and simply learn to identify stimuli as individuals.)

An analytic strategy was also implicit in Gibson's (1969) assertion that perceptual learning involves an active search aimed at discovering attributes that discriminate among stimuli that are to receive different responses. As for category learning, analytic discrimination learning is likely to begin with a search for attributes that would be highly salient, independent of any training. The search would end with the high-salience attributes if they proved to be distinctive to the to-be-discriminated stimuli. Under such circumstances, it would be difficult to empirically demonstrate that subjects were searching for or discovering anything. Evidence that perceptual learning involves searching for and discovering discriminative attributes would be more readily obtained if the analytic strategy persisted and the search process continued beyond testing the attributes that were most salient prior to training. The subject would then have the opportunity to discover discriminative attributes that had been relatively low in salience prior to training.

Previous research concerned with perceptual learning has focused on learning to discriminate among stimuli that were initially difficult to tell apart. At issue in this earlier research was whether improvements in discriminability are the result of predifferentiating individual stimuli by associating different responses with each, as argued by Ellis (1973), or whether they are due to increases in the perceiver's sensitivity to distinct characteristics of the stimuli, as argued by Gibson and Gibson (1955). The present study differs from those experiments, which were concerned with increases in the salience of discriminative attributes. Instead, it is concerned with perceptual 
ing that increases the salience of attributes that are shared by members of the same category.

Evidence consistent with the hypothesis that category learning can increase the salience of attributes shared by members of the same category has been obtained by Homa, Rhoads, and Chambliss (1979). They found, as a result of category learning, that pairs of stimuli belonging to the same category increased in judged similarity, whereas pairs belonging to contrasting categories decreased in judged similarity. However, Homa et al.'s (1979) study provided only indirect evidence for perceptual learning; since they were not concerned with perceptual learning, they made no attempt to identify specific attributes that increased in salience as a result of category learning. Also consistent with the hypothesis that category learning can increase the salience of attributes shared by members of the same category are the results of experiments reported by Medin and Smith (1981) and Medin et al. (1983). In these experiments, changes in attribute salience were indicated by differences in the parameter values that provided the best fit of Medin and Schaffer's (1978) computational formula to their categorization data. Since different training procedures resulted in different parameter values, it could be inferred that at least one of the training procedures changed the salience of the attributes relative to their precategorical values. However, since Medin and his colleagues were not concerned with perceptual learning, they did not assess the precategorical salience of the attributes. Thus, the relative salience of the attributes they tested may have changed as a result of category learning, but there was no way of determining whether the change constituted an increase in salience without also assessing the precategorical salience of the attributes.

Although the issue is fundamental to any theory of categorization, no published evidence, to our knowledge, reports a direct test of whether perceptual learning, in the form of increased salience of attributes shared by members of the same category, can occur as a consequence of category learning. The outcome of such an experiment is by no means obvious. To observe an increase in the salience of shared attributes, it is necessary to begin with a set of stimuli for which attention to precategorically salient attributes is not a sufficient basis for successful category acquisition. Having done so, there is no guarantee that there will be an increase, relative to precategorical values, in the salience of attributes shared by members of the same category. As indicated earlier, subjects may test hypotheses regarding the attributes that are precategorically salient, and when that fails, adopt an item-learning strategy instead of searching for nonobvious, diagnostic attributes (Brooks, 1978).

Since previous research has indicated that the nature of the training procedure can influence whether subjects abstract diagnostic attributes during category learning (Brooks, 1978; Medin et al., 1983), two different acquisition procedures were used in Experiment 1: conceptformation and paired-associate training. The procedures differed in that only the latter required learning to discriminate among members of the same category. We were interested in whether perceptual learning (i.e., increased salience of attributes shared by members of the same category) would occur with a training procedure that encouraged category learning, but required item learning (paired-associate training; each pattern received a different name, but members of the same category had semantically related names), as well as a training procedure that emphasized an analytic strategy by instructing subjects to look for relations among the patterns that were relevant to their category membership (concept-formation training; patterns belonging to the same category received the same name). We included classification and recognition tests following category training in order to evaluate performance differences that were anticipated for the two training procedures.

\section{EXPERIMENT 1}

The stimuli used in this experiment were dot patterns. The attribute structures of these patterns were examined by having subjects segment the patterns into subunits (parts) by drawing circles around groups of dots within each pattern. The parsing procedure we used was similar to procedures used previously by Banks and Prinzmetal (1976) and Palmer (1977). The ability of these investigators to predict performance in various tasks on the basis of how their stimuli were segmented into parts indicated that the parsing procedure successfully reflects important aspects of a stimulus's attribute structure. Furthermore, Tversky and Hemenway (1984) showed that the identifiable parts of an object are critical to the representation of basic level categories for natural objects. These studies demonstrate that the constituent parts of a visual stimulus are empirically and "ecologically" valid units of analysis.

The stimulus attributes that were relevant to our study of perceptual learning were the characteristics of the parts into which the patterns were parsed. The particular characteristics we examined were the number of dots constituting each part, the shape of each part, and the orientation of each part.

The general design of the experiment was to compare how dot patterns were parsed into subunits for three groups of subjects. One group had no category training, one group received concept-formation training, and one group received paired-associate training. We hypothesized that when subjects learned to categorize the patterns, the attributes that were common to patterns that were potential members of the same category would increase in salience relative to their salience in the no-training condition. To show that increases in the salience of attributes shared by members of the same category did not occur by chance (if an attribute becomes more salient, its likelihood of reappearing in the parsing data for many different patterns would also increase), we contrasted the salience of attributes common to patterns drawn from the same category with the salience of attributes common to sets 


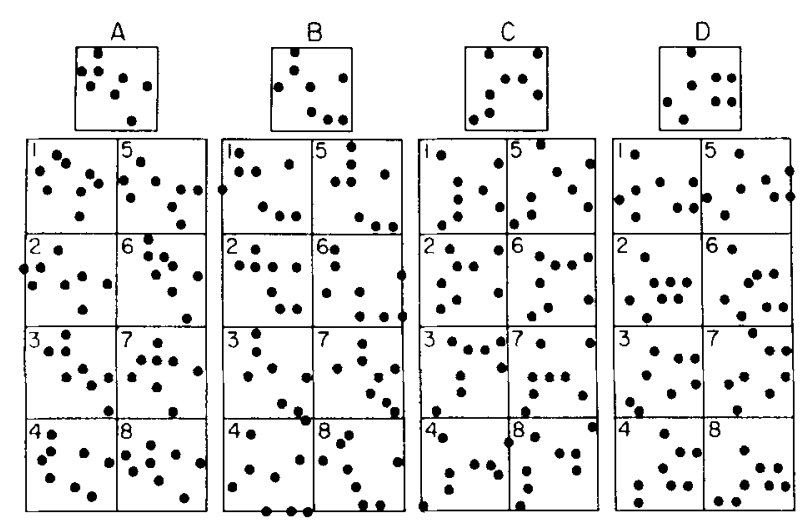

Figure 1. The four base patterns and the eight members of each category that were generated from these base patterns.

of patterns drawn from different categories. Our method of computing common-attribute salience involved a version of Medin and Schaffer's (1978) multiplicative combination rule. We first measured the salience of an attribute as it appeared in each pattern by counting the number of subjects who circled the pattern part when they parsed the pattern. We then determined the common-attribute salience for a set of patterns (i.e., how strongly they resembled each other on the basis of this attribute) by computing the product of the salience values for all the patterns in the set.

\section{Method}

Subjects. A total of 192 subjects provided the data for this experiment: 64 were in the no-training condition, 64 received conceptformation training, and 64 received paired-associate training. Some of the 64 subjects in the no-training condition were tested in large groups (they were not paid); others were tested individually (they received $\$ 2$ ). The 128 subjects in the two training conditions were tested individually and were paid $\$ 2$ per hour for their participation in an experimental session lasting between 1 and $1 \frac{1 / 2}{h}$. All subjects were undergraduate students at Florida Atlantic University, and their participation was voluntary. They had no previous experience with the patterns or foreknowledge that the research was concerned with category learning.

Stimuli. Dot patterns were defined as belonging to the same category if they were generated from the same base pattern by the same procedure. The four base patterns used to generate the categories are presented in the top row of Figure 1. Each base pattern comprised eight dots whose locations were defined by positions on an imaginary $11 \times 11$ grid. The spacing between grid lines was equal to the diameter of one dot, resulting in patterns that were relatively compact. Category members were generated from the base patterns by partitioning the eight dots composing each base pattern into two groups of three dots and one group of two dots. The dots within each group were then moved one space in the same direction, but the different groups were each moved in different directions. Each pattern generated in this way involved a different partition of the base pattern's eight dots into groups of three, three, and two, and a different pattern of movement direction for the three groups. The set of eight patterns generated for each base pattern is presented in Figure 1. The procedure for generating the patterns is illustrated in Figure 2. For descriptive convenience, each category is identified by a letter $(A, B, C$, or D) and each member of a category by a number ( 1 through 8 ). Subset $1-4$ of each category comprised Patterns 1, 2, 3, and 4; Subset 5-8 of each category comprised Patterns 5, 6, 7, and 8 .

Ratings of similarity for all the pairwise combinations (withincategory and between-categories) of Patterns 1,2, 7, and 8 of each category were obtained from 6 subjects who did not participate in any other aspect of the experiment. Their ratings indicated that patterns belonging to the same category resembled each other only slightly more than patterns belonging to different categories. On a scale of 1 (least similar) to 10 (most similar), the mean ratings were 6.6 for pairs of patterns from the same category and 5.1 for pairs of patterns from different categories.

Design. As indicated earlier, there were three groups of subjects in the experiment. The group assigned to the no-training condition had no experience with the patterns prior to parsing them into subunits; the parsing data constituted their only contribution to the experiment. Since these subjects had no idea that the patterns they saw belonged to categories, their data reflected attributes that were salient independent of the category membership of the patterns. The
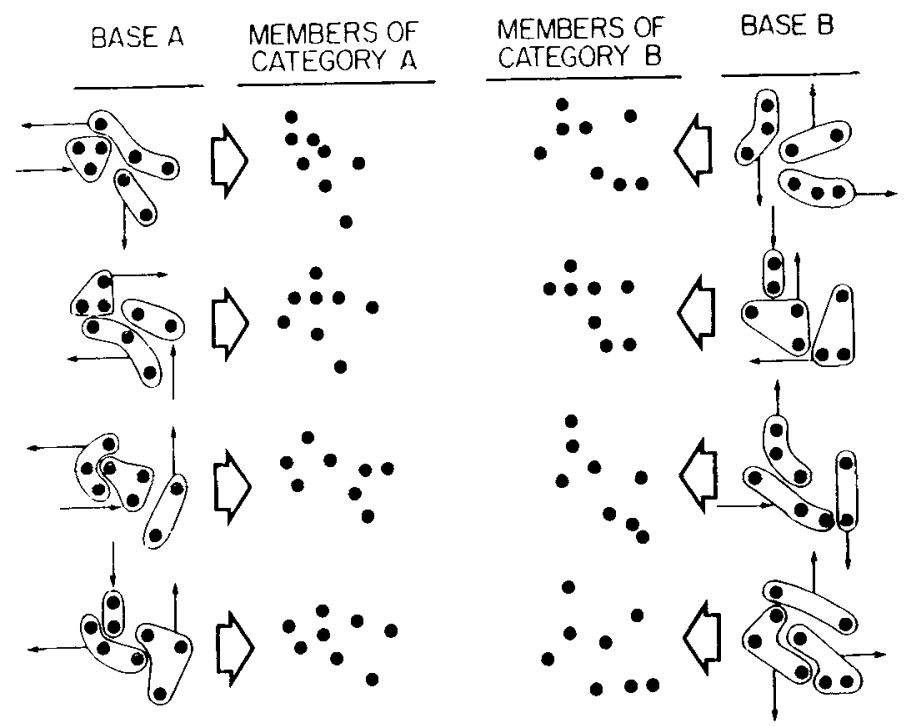

Figure 2. The procedure for generating the membership of each category. 
subjects in the two training conditions participated in an experimental session with three phases: (1) category learning (with either conceptformation or paired-associate training), (2) classification/recognition testing for previously learned and novel category members, and (3) the parsing of four patterns, each from a different category, into their constituent subunits.

For each of the two training conditions, half the subjects learned to identify the patterns in Subset 1-4 of each category, and half learned to identify the patterns in Subset 5-8 of each category. Classification/recognition testing was introduced immediately after acquisition. Thirty-six patterns were presented: 16 were the original exemplar patterns that had been presented numerous times during acquisition, 16 were novel category members that had not been seen before, and 4 were the base patterns that also had not been seen before. If Subset 1-4 comprised the originals during acquisition, Subset 5-8 comprised the novels during classification/recognition testing, and vice versa. The 36 patterns were presented to the subjects in one of two random sequences. Matching sequences were provided for the counterbalancing of Subsets 1-4 and 5-8 as originals and novels. Half the subjects received only recognition testing; the other half received a combination of classification and recognition testing (the purpose of this contrast is unrelated to this paper).

Following classification/recognition testing, the subjects in the training conditions parsed four of the patterns that were not members of the acquisition set. The posttraining parsing data were obtained for novel category members to strengthen the conclusion that changes in attribute salience as a result of category learning would reflect the structure of the categories rather than attributes specific to previously learned patterns. If the subjects had been trained with Subset 1-4 of each category during the acquisition phase of the experiment, they subsequently parsed Patterns A7, B7, C7, and D7 (in counterbalanced order), or Patterns A8, B8, C8, and D8 (also in counterbalanced order). If they had been trained with Subset 5-8 of each category, they subsequently parsed Patterns A1, B1, C1, and D1, or Patterns A2, B2, C2, and D2 (again in counterbalanced order). To ensure that the patterns were properly categorized during the parsing phase, the appropriate category label was typed above each of the to-be-parsed patterns (red, green, blue, and yellow for the subjects who had received concept-formation training; city, color, month, and money for the subjects who had received pairedassociate training). These labels were not provided for the subjects in the no-training condition.

Sixteen subjects provided the parsing data for each of the four packets of patterns (A1B1C1D1, A2B2C2D2, A7B7C7D7, and A8B8C8D8), resulting in a total of 64 subjects in the no-training condition and 64 subjects in each of the two training conditions (concept-formation and paired-associate training). The subjects in both training conditions were assigned to one of eight subgroups defined by the orthogonal combination of parsing set (A1B1C1D1, $\mathrm{A} 2 \mathrm{~B} 2 \mathrm{C} 2 \mathrm{D} 2$, A7B7C7D7, or A8B8C8D8) and intervening testing procedure (recognition or classification + recognition). Eight subjects were assigned to each of these eight subgroups.

Procedure. During the acquisition phase of the experiment, blackon-white slides of the dot patterns were back-projected, using a random-access projector, onto a translucent screen. The size of the patterns on the screen was $2.5 \times 2.5 \mathrm{~cm}$. They were viewed from a distance of approximately $114 \mathrm{~cm}$, thereby intersecting a visual angle of $1.3^{\circ}$. The subjects who received concept-formation training learned the same verbal label for each member of the category (see Table 1). They were told that they would be seeing 16 dot patterns, each of which belonged to one of four groups, and they were instructed to look for relations among the patterns that would allow them to learn which patterns belonged in each group. In pairedassociate training, the subjects learned different verbal labels for each pattern (see Table 1). However, the names for members of the same visual category belonged to the same semantic category. The labels for Category A were all names of colors, those for Category $\mathrm{B}$ were names of cities, those for Category $\mathrm{C}$ were names
Table 1

Assignment of Response Labels to Visual Categories for Concept-Formation and Paired-Associate Training with Either Subset 1-4 or Subset 5-8 of Category Members

\begin{tabular}{|c|c|c|c|c|}
\hline \multirow[b]{2}{*}{ Category } & \multicolumn{4}{|c|}{ Category Members } \\
\hline & $1(5)$ & $2(6)$ & $3(7)$ & $4(8)$ \\
\hline \multicolumn{5}{|c|}{ Concept-Formation Training } \\
\hline $\begin{array}{l}\text { A } \\
B \\
C \\
D\end{array}$ & $\begin{array}{l}\text { red } \\
\text { green } \\
\text { blue } \\
\text { yellow }\end{array}$ & $\begin{array}{l}\text { red } \\
\text { green } \\
\text { blue } \\
\text { yellow }\end{array}$ & $\begin{array}{l}\text { red } \\
\text { green } \\
\text { blue } \\
\text { yellow }\end{array}$ & $\begin{array}{l}\text { red } \\
\text { green } \\
\text { blue } \\
\text { yellow }\end{array}$ \\
\hline \multicolumn{5}{|c|}{ Paired-Associate Training } \\
\hline $\begin{array}{l}\text { A } \\
B \\
\text { C } \\
\text { D }\end{array}$ & $\begin{array}{l}\text { red } \\
\text { Paris } \\
\text { penny } \\
\text { April }\end{array}$ & $\begin{array}{l}\text { green } \\
\text { Rome } \\
\text { nickel } \\
\text { June }\end{array}$ & $\begin{array}{l}\text { blue } \\
\text { London } \\
\text { dime } \\
\text { March }\end{array}$ & $\begin{array}{l}\text { yellow } \\
\text { Madrid } \\
\text { dollar } \\
\text { August }\end{array}$ \\
\hline
\end{tabular}

for money, and those for Category D were names of months. The subjects who received paired-associate training did not receive any instruction that directed them to look for relations among the patterns (although the semantic similarity of the response labels for members of the same category could have had the same effect)

Acquisition began when the subjects were shown 16 exemplar patterns in random order. One group was trained with Subset 1-4 of each category, the other with Subset 5-8 of each category. The experimenter provided the label of each pattern orally during this initial presentation. Subsequent blocks of 16 trials were presented in one of five random sequences. The order of these trial-block sequences was varied from subject to subject. The subjects were given up to $10 \mathrm{sec}$ to verbally identify each pattern, at which point they were required to make their best guess. Corrective feedback was provided following incorrect responses. The acquisition phase proceeded in this manner until two consecutive blocks of 16 trials were completed without error.

Classification/recognition testing followed immediately after category training. The subjects were told that they would be seeing some old patterns and some new ones they had not seen before, and that the patterns belonged to the same groups they had already learned. Those receiving only recognition testing were required to indicate whether or not they had previously seen each pattern. Those who received a combination of classification and recognition testing had to first categorize each test pattern and then indicate whether or not they had seen it before (they were provided with the appropriate category names on an index card). Each pattern was presented, again using a random-access slide projector, for as long a period of time as the subjects needed to respond. They were required to guess if they were unsure of the correct response(s). No corrective feedback was provided.

During the parsing phase, each subject was given a single booklet comprising four $14 \times 20-\mathrm{cm}$ sheets of white paper. The four patterns parsed by each subject came from different categories to avoid the possibility that subjects would feel constrained to repeat parsings should they recognize a pattern to be similar to a previously parsed pattern. One pattern $(2.5 \times 2.5 \mathrm{~cm})$ was presented on each sheet.

Parsing instructions. The subjects were instructed to draw circles around the dots in each pattern to form three clusters (the number of clusters was restricted so that each subject's data would have equal weight in determining the perceptual subunits for each pattern). The subjects who parsed the patterns after either conceptformation or paired-associate training were told that their clusters should reflect the ways in which each pattern resembled other members of its category. The subjects in the no-training condition were told that their clusters should reflect their perception of the natural groupings of dots within the pattern. For both the no-training and 
training conditions, the clusters the subjects formed could overlap, and one cluster could be contained within another. Every dot had to appear in at least one circle, even if it appeared alone, and no circle could surround all the dots in the pattern.

Parsing analysis. The perceptual subunits (i.e., the pattern parts) that were circled by the subjects in parsing each pattern were analyzed in the same way for the subjects in the no-training and training conditions. Each subject-selected subunit was coded according to the attributes of number, orientation, and shape. Number refers to the number of dots within the subunit (1-7). Based on our assumption that it would be a psychologically important attribute only when the subunit was relatively large, the orientation of the subunit was coded only when the two most distant dots were separated by a distance of at least six dot diameters (which was more than half of every pattern's height and width). The orientation, expressed in degrees, was computed from the slope of the line defined by the two most distant dots in the subunit. The alternative shape codes used to describe each subunit included the following: linear (vertical, horizontal, left diagonal, and right diagonal), triangular (obtuse, acute, and right), parallelogram, rectangular, and Y-shaped arrangements.

The salience and relative location of each subunit were used to compute the common-attribute salience for a set of patterns. The salience of each subunit (part) was determined by the number of subjects who circled the subunit. The relative location for each subunit was determined as follows: (1) the centroid was computed for the pattern comprising the subunit; this was the average $x, y$ location of the eight dots composing the pattern, and (2) the relative location of each subunit within its pattern was computed as the average $x, y$ distance of the dots composing the subunit from the centroid of the entire pattern. ${ }^{1}$

The first step in the analysis of common-attribute salience was to pool the parsing data from all the subjects who parsed a particular pattern. That is, for each of the 16 patterns that were parsed, we combined the subunits circled by the 16 subjects (per condition) who parsed the pattern. For each pattern, therefore, we generated a list of subunits, each coded according to number, orientation, shape, relative location, and salience (subunits that were circled by more than one subject were listed once and given a salience value based on the frequency with which they were circled). When our computer program tested for attribute matches among a set of patterns, the matches were based on a comparison of number, orientation, and shape attributes across each of these subunit lists. Sets of four different patterns were selected, some from the same category and some from different categories, and the program determined whether there was an attribute match among the subunits of each of the four patterns. This was done independently for the attributes of number, orientation, and shape; when subunits of different patterns were compared for one of these attributes, the values of the other attributes were ignored. Matches based on number were obtained when there were four subunits, one in each of the four patterns, that had the same number of dots. Matches based on orientation were obtained when there were four subunits, one in each of the four patterns, that had orientations within $45^{\circ}$ of each other. Matches based on shape were obtained when there were four subunits, one in each of the four patterns, that had the same shape code.

The location of subunits within each pattern introduced the problem of criterion. For example, if each pattern in a set of four patterns had a subunit with the same shape, how close in location would the subunits have to be in order to be considered as matching? Our solution was to systematically vary the criterion used to determine whether matching subunits in a set of patterns were in the same relative location within their respective patterns. Thus, the presence of matching subunits was tested when the subunits had to differ in relative location by less than one dot diameter, by less than two dot diameters, and so on, in order to be considered as matching in either number, orientation, or shape. When subunit matches were obtained for one of these attributes, and the four matching subunits were in the same relative location within their patterns (based on the relative-location criterion), common-attribute salience was computed by multiplying the frequency with which each of the four matching subunits was circled when subjects parsed the four patterns. For example, if four of the patterns belonging to Category A (Patterns 1, 2, 7, and 8) had a rectangular subunit at the same relative location, the salience of this common attribute was determined by computing the product of how frequently the subunit was circled for each pattern $\left(f_{1} \times f_{2} \times f_{7} \times f_{8}\right)$. Since the same four patterns could have another shape in common (e.g., subunits forming right triangles), the total common-attribute salience for the attribute of shape was the sum of the frequency products for each shape that was common to all four patterns. Finally, the fourth root of the summed frequency products was computed in order to place the computation of common-attribute salience on a scale that would reflect the relative salience of the matching subunits within each of their patterns (the maximum value for each subunit, 16 , would be obtained if the subunit common to all four patterns was circled by all 16 subjects for each of the patterns).

The above analysis was performed for eight sets of four patterns. The analysis for the first four sets, A1A2A7A8, B1B2B7B8, $\mathrm{C} 1 \mathrm{C} 2 \mathrm{C} 7 \mathrm{C} 8$, and D1D2D7D8, assessed common-attribute salience for patterns belonging to the same category (recall that each subject parsed only one pattern from each of the four categories). The analysis for the second four sets, A1B2C7D8, A2B7C8D1, $\mathrm{A} 7 \mathrm{~B} 8 \mathrm{C} 1 \mathrm{D} 2$, and $\mathrm{A} 8 \mathrm{~B} 1 \mathrm{C} 2 \mathrm{D} 7$, assessed common-attribute salience for patterns belonging to different categories. The latter four sets of patterns represent only a sample of all possible between-categories combinations. In selecting this sample, we avoided betweencategories combinations corresponding to the packets of four patterns presented to subjects in the parsing task (A1B1C1D1, $\mathrm{A} 2 \mathrm{~B} 2 \mathrm{C} 2 \mathrm{D} 2, \mathrm{~A} 7 \mathrm{~B} 7 \mathrm{C} 7 \mathrm{D} 7$, and $\mathrm{A} 8 \mathrm{~B} 8 \mathrm{C} 8 \mathrm{D} 8$ ). For each attribute tested, we contrasted within-category and between-categories common-attribute salience for the no-training, concept-formation, and paired-associate conditions. ${ }^{2}$

\section{Results}

A number of different data analyses are reported in this section. The primary analysis is of the parsing data, which provides our basic evidence for the effect of category learning on the salience of attributes shared by patterns belonging to the same category. Preceding the analysis of the parsing data are analyses of acquisition rates, postacquisition recognition accuracy, and postacquisition classification accuracy.

Acquisition. All 128 subjects in the training conditions reached our criterion of two errorless blocks of 16 trials. The subjects who received concept-formation and pairedassociate training required an average of 14.4 and 8.0 trial blocks (excluding the two errorless criterion blocks), respectively, to reach the acquisition criterion. The advantage of paired-associate training was statistically significant $[t(126)=5.46, p<.001]$. We also compared the two training conditions with regard to the proportion of errors within each block of 16 trials. To control for differences in response confusability in the two training conditions (there were 16 different responses in the pairedassociate condition, compared with 4 in the conceptformation condition), the paired-associate data were scored only for between-categories acquisition errors (e.g., if a subject responded "nickel"' to a pattern that 
should have been called "dime," he/she was told that the response was wrong, but we subsequently scored it as correct). The mean error percentage rates per trial block (all between-categories errors) for the two training conditions are presented in Figure 3. An analysis of variance based on the first 10 trial blocks indicated that fewer errors were made in paired-associate than in the concept-formation training $[F(1,126)=506.54, p<.001, M S \mathrm{e}=$ 1568.87]. The significant interaction between training procedure and trial block $[F(9,1134)=7.04, p<.001$, $M S e=114.84]$ reflected the emergence of the pairedassociate advantage in category learning after the first block of acquisition trials. Because the two training procedures differed with regard to the need for individual item learning, it could be concluded that the paired-associate advantage resulted from enhanced item learning. The proportion of all errors during paired-associate training that were within the same category (e.g., calling a pattern "Rome" when the correct response was "Madrid") started at $29 \%$ for the first block of trials, and gradually increased as practice proceeded. This rate of withincategory errors was consistently higher than the $20 \%$ rate that would have occurred strictly by chance.

Classification/recognition testing. The results of this testing phase, which was interposed between the training and parsing phases of the experiment, are summarized in Table 2 . The recognition data were analyzed by converting hits and false alarms into $d^{\prime}$ scores for each subject. All 128 subjects who received the recognition test discriminated novel category members from previously

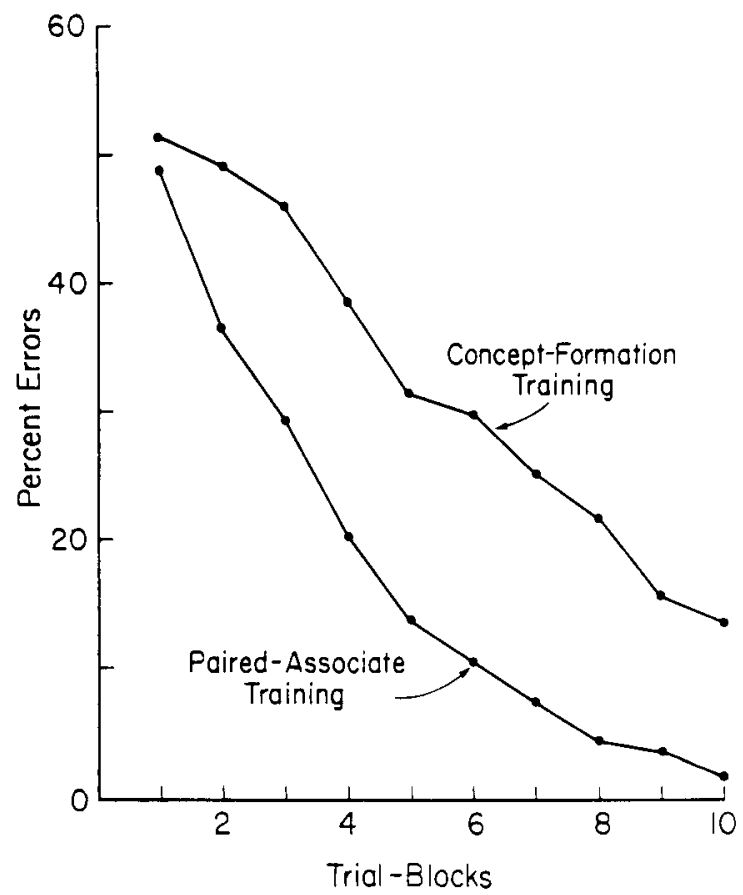

Figure 3. Experiment 1: The percentage of between-categories errors, per trial block, during concept-formation and paired-associate training.
Table 2

Percent Classification and Recognition Errors for Previously

Seen (Original), Novel, and Base Patterns Following Either Concept-Formation or Paired-Associate Training in Experiment 1

\begin{tabular}{lcc}
\hline $\begin{array}{c}\text { Test } \\
\text { Patterns }\end{array}$ & $\begin{array}{c}\text { Classification } \\
\text { Errors }\end{array}$ & $\begin{array}{c}\text { Recognition } \\
\text { Errors }\end{array}$ \\
\hline \multicolumn{3}{c}{ Concept-Formation Training } \\
Originals & 12.5 & 56.7 \\
Novels & 5.1 & 10.9 \\
& 32.4 & 20.2 \\
& Paired-Associate Training \\
Bases & 15.6 & 39.5 \\
Originals & 3.7 & 7.4 \\
Novels & 34.0 & 14.3 \\
\hline
\end{tabular}

seen category exemplars at a better than chance rate. A comparison of the two training procedures indicated that $d^{\prime}$ scores (based on hits for the original, exemplar patterns and false alarms for the novel patterns) were significantly better in the paired-associate (mean $=2.9$ ) than the concept-formation condition (mean $=2.5$ ), $[t(126)$ $=3.35, p<.002]$. The subjects who received pairedassociate training also made significantly fewer falserecognition responses on the base patterns than did subjects who received concept-formation training $[t(126)=$ $3.54, p<.001]$. It should be noted that superior recognition performance was obtained for the paired-associate condition even though the subjects received fewer exposures to the original, exemplar patterns during training than did subjects in the concept-formation condition.

The classification data were very similar for the concept-formation and paired-associate conditions. An analysis of variance on percent classification errors indicated that the original/novel difference was significant $[F(1,62)=202.95, p<.001, M S e=130.85]$, but the effect of training procedure $[F(1,62)<1.0, M S e=$ 187.49] and the interaction between pattern type (original/novel) and training procedure $[F(1,62)<1.0, M S \mathrm{e}$ $=130.85]$ were not significant. Although classification accuracy was much lower for the novel than the original category members, the subjects' classification of the novels (33.2\% error rate) was nonetheless much better than chance ( $75 \%$ error rate). All 64 subjects classified the novels at better than chance rates. The significance of these data is that when the subjects went on to the parsing phase of the experiment, their ability to categorize the novel patterns was very similar following conceptformation and paired-associate training.

Parsing. Our initial examination of the parsing data was concerned with the extent to which there was agreement regarding the subunits circled by each subject. We assessed intersubject agreement by counting the number of different subunits circled by all of the 16 subjects who parsed each pattern and comparing this number to the number of different subunits that would have been circled had there been no agreement among the subjects $(3 \times 16=48)$. If all subjects were in complete agreement, 
the same three subunits would have been circled by each subject, indicated by an agreement factor of $.06(3 / 48)$; no agreement would be indicated by an agreement factor of $1.00(48 / 48)$. We found that category learning resulted in decreased levels of intersubject agreement relative to the no-training condition. The agreement factor was .35 for subjects in the no-training condition, .45 for subjects who received concept-formation training, and .46 for subjects who received paired-associate training. The increase in intersubject variability was observed to be consistent for all 16 patterns that were tested; a reversal was obtained for only one pattern, and then only following concept-formation training.

As indicated in the Method section, our criterion for determining whether a subunit was large enough for its orientation to be a psychologically important variable was that the two most distant dots in the subunit were separated by at least six dot diameters (more than half of the height and width of every pattern). In comparing the parsing data for the no-training and training conditions, we found that large subunits were more salient for subjects in the training conditions compared with subjects in the no-training conditions. The percentages of subunits that were large by this criterion were $19.0 \%, 30.9 \%$, and $31.0 \%$, for the no-training, concept-formation, and paired-associate conditions, respectively. With individual patterns serving as the random variable, an analysis of variance indicated that the effect of training condition (notraining, concept-formation, paired-associate) on the percentage of large subunits in a pattern was significant $[F(2,24)=15.70, p<.001, M S e=48.22]$, and fur- thermore, that the interaction between the training procedure and the category to which the patterns belonged $(\mathrm{A}, \mathrm{B}, \mathrm{C}, \mathrm{D})$ was not significant $[F(6,24)<1.0, \mathrm{MSe}=$ 48.22]. That is, the effect of the training condition on subunit size was similar for all four categories used in the experiment. Newman-Keuls comparisons indicated that the physical size of the subjects' subunits was significantly larger following either concept-formation or paired-associate training compared with the no-training condition $(p<.05) .^{3}$

In addition to increasing the salience of large subunits, category learning increased the likelihood that patterns that belonged to the same category would share large subunits that were similar in orientation. The latter was assessed in terms of common-attribute salience. The computation of orientational common-attribute salience for a set of patterns required that all the patterns in the set contain large subunits with orientations within $45^{\circ}$ of each other. The computations were performed for sets of four patterns (e.g., A1A2A7A8) from the same category, and sets of four patterns (e.g., A1B2C7D8) from different categories (see Method section for details). As can be seen in Figure 4, common-attribute salience increased in all conditions as the relative-location criterion was relaxed. The looser relative-location criteria allowed more subunits to be considered as matching than did the more stringent criteria. Figure 4 also indicates that common-attribute salience increased, relative to the no-training condition, as a result of both concept-formation and paired-associate training. Increases were observed for sets of four patterns from the same category (within-category) as well as for
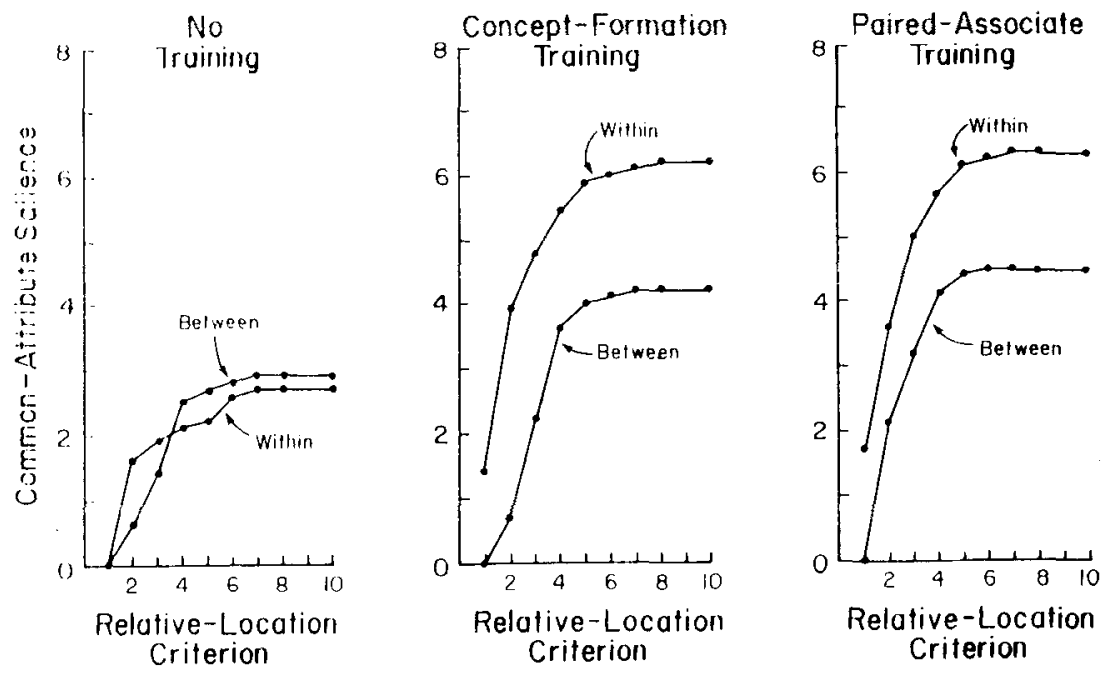

Figure 4. Experiment 1: Common-attribute salience for sets of four patterns that were members (or potential members) of the same category (within) or different categories (between). Communality was based on corresponding subunits' being similar in orientation and being in the same relative location for all four patterns (the latter was determined over a wide range of relative-location criteria). Subjects in the no-training condition looked for "perceptually natural" subunits in parsing each pattern. Subjects in the concept-formation and paired-associate conditions looked for subunits that reflected resemblances with other category members in parsing each pattern. 
sets of four patterns from different categories (betweencategories). The increase in between-categories commonattribute salience as a result of category learning could be attributed to the increased prevalence of large subunits, which increased the likelihood of large subunits of similar orientation occurring by chance. Most important, common-attribute salience for patterns from the same category was greater than the baseline/chance level observed for patterns from different categories for the subjects in the two training conditions, but there was no difference from baseline for the subjects in the no-training condition. ${ }^{4}$

An analysis of variance was performed, in which the random variable was the set of four patterns analyzed for orientational common-attribute salience. There were four sets of four patterns in the within-category condition (A1A2A7A8, B1B2B7B8, etc.) and four sets of four patterns in the between-categories condition (A1B2C7D8, A2B7C8D1, etc.). The analysis of common-attribute salience indicated that the interaction between training condition (no-training, concept-formation, pairedassociate) and the type of pattern set (within-category vs. between-categories) was significant $[F(2,18)=16.38$, $p<.001, M S e=5.18]$. This interaction was not significantly affected by the relative-location criterion used to determine whether there were matching subunits within each set of four patterns tested. That is, the three-way interaction between training condition, type of pattern set, and relative-location criterion was not significant $[F(16,144)<1.0, M S e=0.23]$. Tests of simple effects indicated that the effect of type of pattern set (withincategory vs. between-categories) on common-attribute salience was significant following concept-formation training $[F(1,18)=15.11, p<.002, \mathrm{MSe}=5.18]$, and was also significant following paired-associate training $[F(1,18)=10.13, p<.02, M S e=5.18]$, but was not significant in the no-training condition $[F(1,18)<1.0$, $M S e=5.18]$. Finally, the overall effect of training condition on common-attribute salience was significant $[F(2,18)=22.42, p<.001, M S e=5.18]$, and subsequent Newman-Keuls comparisons indicated that common-attribute salience was not statistically different in the concept-formation and paired-associate conditions $(p>.05)$ and also, that both training conditions resulted in higher levels of common-attribute salience than the notraining condition $(p<.05)$.

\section{Discussion}

Although large subunits increased in salience as a result of category learning (relative to the no-training condition), we did not consider this sufficient evidence for perceptual learning. The increased salience of large subunits could have been due to a general learning strategy elicited by our category training procedures; there may have been a bias to emphasize large pattern parts. Stronger evidence for perceptual learning would be obtained if it could be demonstrated that increases in attribute salience were specific to the pattern information that was acquired during category training. Our primary evidence for percep- tual learning was therefore based on the orientational similarity of subunits (parts) of patterns that belong to the same category. As a result of category training, we obtained an increase in the salience of large subunits that were similar in orientation for patterns that belonged to the same category; a comparable increase was not obtained for patterns that belonged to different categories. In concluding that we have evidence for perceptual learning, we are not arguing that the subjects' perception of the natural grouping of elements in a pattern has changed. Rather, our claim is that we have detected an attribute, common to members of the same category, whose likelihood of being noticed increased as a result of category learning.

\section{EXPERIMENT 2}

In this experiment, we investigated the extent to which the results obtained in Experiment 1 depended on extended category training rather than differences in parsing instructions between the no-training and training conditions. The subjects in Experiment 2 participated in one of two conditions that differed with regard to the requirements of the parsing task: a similarity condition and a difference condition. Like the no-training condition of Experiment 1 , the parsing task in these conditions was not preceded by extended category training, and the subjects had no knowledge of the category structure of the patterns. Nonetheless, the parsing instructions reflected potentially important aspects of category structure. Thus, the subjects in the similarity condition were told to circle groupings of dots in each pattern that made the four patterns in a packet look similar to each other (the patterns were potential members of the same category), whereas the subjects in the difference condition were told to circle groupings of dots in each pattern that made the four patterns in a packet look different from each other (the patterns were potential members of different categories). ${ }^{5}$ Although they did not receive the extended category training provided in the concept-formation and paired-associate conditions of Experiment 1, grouping the patterns into packets gave the subjects the opportunity for rapid category learning by eliminating the need for exemplar retrieval while they were looking for shared or discriminative attributes (in contrast, patterns were presented one at a time during the training sessions of Experiment 1). Thus, the subjects did not have to retrieve previously seen patterns to look either for attributes that were shared by patterns that were potential members of the same category or for attributes that differentiated patterns that were potential members of different categories; the patterns that the subjects compared were presented together in the same packet.

By focusing on the discovery of shared/discriminative attributes, the experiment allowed us to determine the extent to which the orientational attributes discovered by the category learners in Experiment 1 were precategorically salient. It would not be surprising if subjects in this experiment discovered some of these attributes, especially 
in the similarity condition, which allowed for the direct comparison of patterns from the same category. If all the orientational attributes discovered by the category learners of Experiment 1 were precategorically salient, the contrast in orientational common-attribute salience for withincategory compared to between-categories pattern combinations obtained in this experiment would be similar to that obtained for the training conditions of Experiment 1. If this was not the case, despite maximizing the opportunity for the subjects to notice shared attributes, it could be concluded that extended training was required for the discovery of at least some of the orientational resemblances among members of the same category.

\section{Method}

Subjects. One hundred and twenty-eight subjects, all tested in large groups, provided the data for this experiment. Half were assigned to the difference condition and half to the similarity condition. All were undergraduate students at Florida Atlantic University who had no previous experience with the patterns or foreknowledge that the research was concerned with category learning.

The difference condition. The packets of four patterns given to the subjects were the same as in Experiment 1; each of the patterns in a packet came from a different category. Although the general parsing instructions were the same as in Experiment 1, they differed in that the subjects were now instructed to circle groups of dots in each pattern that made the four patterns in each packet look different from each other. They were told to examine all the patterns before beginning to circle groups of dots for the first pattern in the packet.

The similarity condition. In contrast with the difference condition and all three conditions of Experiment 1, the packets of four patterns given to the subjects all came from the same category. There were 16 packets comprising patterns A1A2A7A8, 16 comprising patterns $\mathrm{B} 1 \mathrm{~B} 2 \mathrm{~B} 7 \mathrm{~B} 8,16$ comprising patterns $\mathrm{C} 1 \mathrm{C} 2 \mathrm{C} 7 \mathrm{C} 8$, and 16 comprising patterns D1D2D7D8. The four patterns in each set were presented in four different, counterbalanced orders. The subjects were instructed to circle groups of dots in each pattern that made the four patterns in each packet look similar to each other. They were told to examine all the patterns before beginning to circle groups of dots for the first pattem in the packet.

\section{Results}

The difference condition. Intersubject agreement was similar to that observed for the training conditions of Experiment $1(.44$ in this experiment compared with .45 and .46 for the concept-formation and paired-associate conditions of Experiment 1). The salience of large subunits was similar to that obtained in the no-training condition of Experiment $1 ; 22.4 \%$ of the subunits were relatively large, compared with $19.0 \%$ in the no-training condition. Overall levels of orientational common-attribute salience were greater than the levels observed for the no-training condition of Experiment 1, but there was no difference in common-attribute salience for sets of patterns drawn from the same category and sets of patterns drawn from different categories (see Figure 5). An analysis of variance indicated the type of pattern set (within-category vs. between-categories) did not significantly affect commonattribute salience $[F(1,6)<1.0, M S e=4.94]$. Further-

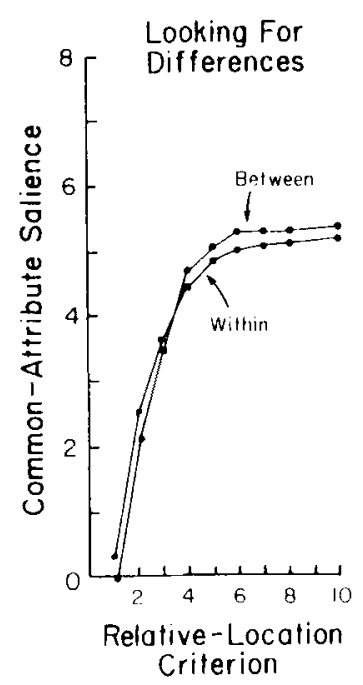

Figure 5. Experiment 2: Common-attribute salience for sets of four patterns that were potential members of the same category (within) or different categories (between). Communality was based on corresponding subunits' being similar in orientation and being in the same relative location for all four patterns (the latter was determined over a wide range of relative-location criteria). Subjects looked for subunits that reflected differences among the four patterns that they parsed.

more, this result was not influenced by the relativelocation criterion; the interaction between the type of pattern set and the value of the relative-location criterion was not significant $[F(8,48)<1.0$, MSe $=0.22]$.

The similarity condition. The level of agreement regarding the subunits circled by each subject (the agreement factor was .41) was intermediate to the agreement factors obtained in the no-training and training conditions of Experiment 1. The salience of large subunits was again similar to that obtained in the no-training condition of Experiment $1 ; 22.4 \%$ of the subunits were relatively large, compared with $19.0 \%$ in the no-training condition. Despite the relatively low salience of large subunits, overall levels of orientational common-attribute salience were similar to those obtained for the training conditions of Experiment 1 , and common-attribute salience was greater for sets of patterns from the same category compared with sets of patterns from different categories (see Figure 6). This difference, however, was not reliable. An analysis of variance indicated that the type of pattern set for which attribute matches were obtained (within-category vs. between-categories) did not significantly affect commonattribute salience $[F(1,6)=1.34, p>.05, M S e=$ $20.10]$. This result was not significantly influenced by the relative-location criterion; the interaction between the type of pattern set and the value of the relative-location criterion was not significant $[F(8,48)<1.0, M S e=0.55]$.

\section{Discussion}

Large subunits were no more salient in the difference and similarity conditions of this experiment than they were in the no-training condition of Experiment 1 . It was there- 


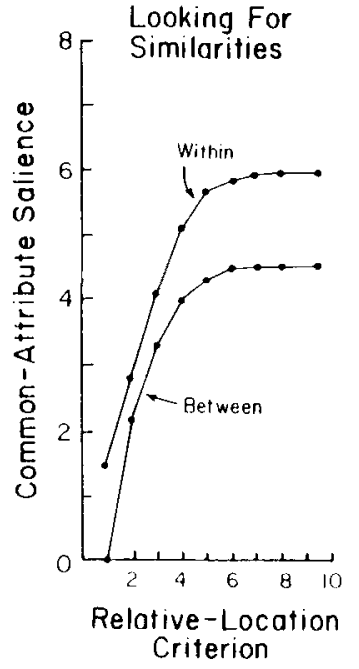

Figure 6. Experiment 2: Common-attribute salience for sets of four patterns that were potential members of the same category (within) or different categories (between). Communality was based on corresponding subunits' being similar in orientation and being in the same relative location for all four patterns (the latter was determined over a wide range of relative-location criteria). Subjects looked for subunits that reflected similarities among the four patterns that they parsed.

fore unlikely that the relatively low salience of large subunits was due to the parsing instructions used in the no-training condition. Only the results obtained in the similarity condition provided evidence suggesting that the orientational attributes discovered by the subjects in the training conditions of Experiment 1 were precategorically salient. However, the absence of a reliable difference in common-attribute salience between patterns drawn from the same category and patterns drawn from different categories indicated that large subunits that were similar in orientation may have been precategorically salient for some categories, but not others.

This was borne out by a category-by-category comparison of the similarity condition of Experiment 2 with the training conditions of Experiment 1 (the results for the

Table 3

Common-Attribute Salience, Averaged Over All Relative-Location Criteria, for Sets of Four Patterns That Were Potential Members of the Same Category (Within Category) and Sets of Four Patterns That Were Potential Members of Different Categories (Between Categories)

\begin{tabular}{ccc}
\hline & $\begin{array}{c}\text { Training Con- } \\
\text { ditions } \\
\text { (Experiment 1) }\end{array}$ & $\begin{array}{c}\text { Similarity Condition } \\
\text { (Experiment 2) }\end{array}$ \\
\hline \multicolumn{3}{c}{ Within Category } \\
A & 5.7 & 3.8 \\
B & 5.3 & 3.0 \\
C & 5.5 & 7.7 \\
D & 4.2 & 4.5 \\
& Between Categories \\
& 3.3 & 3.5 \\
\hline
\end{tabular}

Note-The data are reported by category for the training conditions of Experiment 1 (the concept-formation and paired-associate conditions are combined) and the similarity condition of Experiment 2. concept-formation and paired-associate conditions were combined). As can be seen in Table 3 (we have averaged the common-attribute salience for all values of the relativelocation criterion), orientational common-attribute salience was greater for patterns that were members of the same category than for patterns that were members of different categories (the baseline/chance level) for Categories $\mathrm{C}$ and $\mathrm{D}$ in both the training conditions of Experiment 1 and the similarity condition of Experiment 2. Since extended category training was not necessary for the subjects' parsing data of Categories $C$ and $D$ to be affected by orientation, it is possible that orientation was a precategorically salient attribute for these patterns. However, for Categories A and B, orientational commonattribute salience was greater for patterns from the same category than patterns from different categories for the training conditions of Experiment 1, but not for the similarity condition of Experiment 2. Thus, although the opportunity for subjects in the similarity condition to notice the orientational resemblance of these categories was maximized by putting four patterns from the same category into each subjects' packet, the results were substantially different from those obtained in the training conditions (in which each subject's packet contained patterns drawn from different categories). It can be concluded, therefore, that the extended category training provided in the concept-formation and paired-associate conditions of Experiment 1 was responsible for the increase in subjects' sensitivity to the orientational resemblances among the members of Categories A and B.

\section{GENERAL DISCUSSION}

Our perceptual learning hypothesis was framed in conjunction with an analytic category-learning strategy, which emphasizes the search for attributes that are diagnostic of category membership (Brooks, 1978). Evidence for perceptual learning was obtained under training conditions that emphasized analytic category learning (the conceptformation condition), but was also obtained under train ing conditions that appear to have induced an item-learning strategy (the paired-associate condition). Evidence for the latter came from an examination of the acquisition and recognition data for the two training conditions of Experiment 1 .

The acquisition data indicated that paired-associate training resulted in faster category learning than conceptformation training, despite the former having a more complex stimulus-response mapping than the latter. This result was consistent with previous research indicating that when stimuli that belong to the same category are sufficiently dissimilar, acquisition can benefit from a training procedure that requires the learning of the stimuli as individual items (Brooks, 1978; Medin et al., 1983). Better recognition accuracy (original/novel discrimination) following paired-associate compared with concept-formation training provided further evidence that the former was more facilitative of item learning. Despite these differences, the subjects in the two training conditions were alike with 
regard to their ability to classify previously seen and novel category members. They were also alike with regard to their parsing of the patterns. For both training conditions, patterns belonging to the same category increased in their tendency to share large subunits that were similar in orientation (relative to the no-training condition). Evidence for the abstraction of shared attributes was therefore obtained in both training conditions, even though subjects appear to have adopted an item-learning strategy in the pairedassociate condition.

Although Brooks (1978) characterized abstractive strategies as analytic and item-learning strategies as nonanalytic (and presumably nonabstractive), the results obtained in the paired-associate condition indicate that the abstraction of attributes shared by members of the same category is not empirically incompatible with item learning. In another study, Hock, Tromley, and Polmann (in press) argued that the long-term retention of previously learned category exemplars may be functionally dependent on the abstraction of shared attributes; attributes shared by members of the same category could facilitate the retention of individual patterns by providing organizational links among the memory representations corresponding to the previously seen category members. Also, Hock, Smith, Escoffery, Bates, and Field (1987) found that superficial pattern details that might be expected to be encoded in a nonanalytic, pictorial format, are abstracted from patterns in the same way as attributes shared by patterns belonging to the same category.

The results of these experiments, together with those of the present study, join others (Ward \& Scott, 1987) to suggest that category learning might always be analytic. The category learner may abstract attributes shared by members of the same category and/or attributes that are unique to particular category members. Differences in within-category and between-categories similarity, as well as differences in training procedure, could influence the extent to which the category learner abstracts shared versus item-specific attributes. ${ }^{6}$ Furthermore, both the shared and distinctive attributes could be stored in exemplar format (as argued by Medin \& Schaffer, 1978), or the shared attributes could be stored in separate, central representations (as maintained by investigators going back to Bartlett, 1932). In either case, the information retained would be in the form of abstracted attributes.

Our concluding discussion concerns the issue of intersubject agreement. Measures of intersubject agreement obtained in the present study indicated that perceptual learning took place in the absence of increases in intersubject agreement. This result is of interest because levels of intersubject agreement on the attributes that characterize various objects or concepts has emerged in the literature as an important empirical index of category knowledge (Murphy \& Wright, 1984; Rifkin, 1985; Rosch \& Mervis, 1975; Rosch, Mervis, Gray, Johnson, \& Boyes-Braem, 1976; Tversky \& Hemenway, 1983, 1984). Murphy and Wright's (1984) paper is particularly relevant to the present study. They report the results of an attribute-listing experiment for three diagnostic categories of childhood adjustment problems; subjects were required to provide verbal lists of the attributes of each adjustment problem. Instead of studying the acquisition of these categories, they compared the attribute listings generated by individuals with varying levels of realworld expertise (ranging from supervisors in a residential treatment program to students in undergraduate psychology courses). Murphy and Wright found that intersubject agreement on the characteristic attributes of these disorders increased with higher levels of expertise, but the extent to which these attributes differentiated among the three categories actually decreased with expertise.

Murphy and Wright's (1984) results suggest that there may be a problem with the assumption, implicit in experiments using the attribute-listing technique, that intersubject agreement is the hallmark of category knowledge. Central to this assumption is the expectation that category learning should result in increased intersubject agreement concerning the attributes that are diagnostic of category membership. However, Murphy and Wright found that increased intersubject agreement was not accompanied by increased salience of attributes that discriminate among contrasting categories, and we have found in the present study that an increase in the salience of attributes shared by members of the same category was obtained without an increase in intersubject agreement.

A critical factor affecting changes in intersubject agreement as a result of training may be the number of attributes that are potentially diagnostic of category membership. If intersubject agreement is to increase as a result of category learning, then the number of potentially diagnostic attributes for the category must be relatively small. The larger the number of potentially diagnostic attributes for category learners to choose among, the greater the likelihood that they will disagree regarding the particular attributes they select as diagnostic of category membership. For the dot patterns used in the present study, the number of attributes that were potentially diagnostic of category membership was likely to have been quite large. Each dot, each pair of dots, triplets, and so forth, and the relations among them, could ultimately generate a vast number of attributes, many of which could have been diagnostic of category membership. The presence of so many alternatives would make it unlikely that category learners would select the same attributes in learning the categories. Consequently, their level of intersubject agreement did not increase, and may have decreased as a result of category learning.

Despite the general disagreement among subjects, we obtained evidence that category learning resulted in patterns belonging to the same category sharing parts that were similar in orientation. This evidence was a consequence of there being at least some agreement among subjects. It was not logically necessary for there to have been any agreement; each subject could conceivably have discovered a different set of attributes that were shared by members of the same category. From the point of view 
of the present study, we were fortunate that there was some agreement that emerged despite the tendency toward increased intersubject variability; otherwise we would not have detected the presence of perceptual learning. We were likewise fortunate to have analyzed subunit orientation; otherwise we would not have hit upon the shared attribute discovered by some of our subjects.

In conclusion, it would be reasonable to ask why investigators, beginning with Rosch and Mervis (1975), have been so successful at obtaining high levels of intersubject agreement regarding the attributes of objects, concepts, scenes, and events. It may be that levels of intersubject agreement in these studies have been overestimated because of the verbal-listing technique these investigators used to identify the attributes. We argued previously that the level of intersubject agreement regarding the attributes of a stimulus depends on the number of attributes that were potentially diagnostic of category membership prior to category learning. A second, related factor concerns the extent to which the measurement technique constrains what subjects can indicate about a stimulus's attributes. Although all measurement techniques, including our own, are to some extent constraining, the verbal listing technique may be excessively constraining in that it restricts the subjects to identifying attributes that lend themselves to brief verbal description. As a result, the number of alternative attributes that are verbally associated with an object, concept, scene, or event will be relatively small, and the potential for intersubject agreement is enhanced. Whether or not subjects agree may tell us more about the constraints inherent in the attribute-identification technique than it tells us about their category knowledge.

\section{REFERENCES}

Banks, W. P., \& Prinzmetal, W. (1976). Configurational effects in visual information processing. Perception \& Psychophysics, 19 , 361-367.

BARTLETT, F. C. (1932). Remembering: A study in experimental and social psychology. Cambridge, England: Cambridge University Press.

BrooKs, L. R. (1978). Non-analytic concept formation and memory for instances. In E. Rosch \& B. C. Lloyd (Eds.), Cognition and categorization. New York: Erlbaum.

ELLIs, H. C. (1973). Stimulus encoding processes in human learning and memory. In G. H. Bower (Ed.), The psychology of learning and motivation (Vol. 7). New York: Academic Press.

Fried, L. S., \& HolyoaK, K. J. (1984). Induction of category distributions: A framework for classification learning. Journal of Experimental Psychology: Learning, Memory, \& Cognition, 10, 234-257.

Gibson, E. J. (1969). Principles of perceptual learning and develop ment. New York: Appleton-Century-Crofts.

Gibson, J. J., \& GiBson, E. J. (1955). Perceptual leaming: Differentiation or enrichment? Psychological Review, 62, 32-41.

Hock, H. S., Smith, L. B., Escoffery, L., Bates, A., \& Field, L. (1987). The perception of pattern: Coding the position of component elements. Manuscript in preparation.

Hock, H. S., Tromley, C., \& Polmann, L. (in press). Perceptual units in the acquisition of visual categories. Journal of Experimental Psychology: Learning, Memory, \& Cognition.

Homa, D., Rhoads, D., \& Chambliss, D. (1979). Evolution of conceptual structure. Journal of Experimental Psychology: Human Learning \& Memory, 5, 11-23.
Medin, D. L., Dewey, G. I., \& Murphy, T. D. (1983). Relationships between item and category learning: Evidence that abstraction is not automatic. Journal of Experimental Psychology: Learning, Memory, \& Cognition, 9, 607-625.

Medin, D. L., \& Schaffer, M. M. (1978). Context theory of classification learning. Psychological Review, 85, 207-238.

Medin, D. L., \& SMiтh, E. E. (1981). Strategies and classification learning. Journal of Experimental Psychology: Human Leaming \& Memory, 7, 241-253.

MurPhy, G. L., \& Wright, J. C. (1984). Changes in conceptual structure with expertise: Differences between real-world experts and novices. Joumal of Experimental Psychology: Learning, Memory, \& Cognition, 10, 144-155.

PALMer, S. E. (1977). Hierarchical structure in perceptual representation. Cognitive Psychology, 9, 441-474.

RIFKIN, A. (1985). Evidence for a basic level in event taxonomics. Memory \& Cognition, 13, 538-556.

Rosch, E., \& Mervis, C. B. (1975). Family resemblances: Studies in the internal structure of categories. Cognitive Psychology, 7, 573-605

Rosch, E., Mervis, C. B., Gray, W. D., Johnson, D. M., \& BoyesBraem, P. (1976). Basic objects in natural categories. Cognitive Psychology, 8, 382-439.

Tversky, B., \& Hemenway, K. (1983). Categories of environmental scenes. Cognitive Psychology, 15, 121-149.

TVERSKY, B., \& HemenWaY, K. (1984). Objects, parts and categories Journal of Experimental Psychology: General, 113, 169-193.

WARD, T. B., \& SCOTT, J. (1987). Analytic and holistic modes of learning family-resemblance concepts. Memory \& Cognition, 15, 42-54.

\section{NOTES}

1. We are grateful to John Jonides for his valuable suggestions concerning the methodology used to establish the relative location of matching subunits in different patterns. The location of each subunit was determined relative to the centroid of its pattern to provide a frame of reference that would adjust itself to differences in the distribution of dots within each pattern.

2. We thank Michael Lilie for writing the computer program used to analyze for common-attribute salience in the subjects' parsing data.

3. In addition to being physically larger, the subunits circled by the subjects following category training tended to incorporate more dots than was the case in the no-training condition. Whereas the percentage of subunits with one or two dots remained virtually constant at $30 \%$, the percentage of subunits with four to seven dots increased from $27 \%$ in the no-training condition to $31 \%$ and $36 \%$ in the concept-formation and paired-associate conditions, respectively. The average number of dots in each subunit was relevant to our measurement of intersubject agreement because it affects the chance rate of agreement; increases in the number of dots per subunit reduce the number of alternative ways in which a pattern can be parsed into three subunits (this is the case regardless of whether the subunits overlap, as they could in all conditions). Although this would have biased our results toward greater intersubject agreement for the training conditions, we found that intersubject agreement decreased rather than increased as a result of training. 4. Similar analyses of two other attributes, the number of dots in each subunit and the shape of each subunit, indicated that changes in commonattribute salience as a result of category learning were no different for sets of four patterns drawn from the same category and sets of four patterns drawn from different categories.

5. For the subjects in Experiment 1 who received category training, it was sensible to use parsing instructions that referred to the category structure of the patterns. However, such instructions were inappropriate in the no-training condition of Experiment 1 and both the difference and similarity conditions of Experiment 2 . This was because the subjects' experience with the patterns was limited in the latter three conditions; they saw patterns drawn from either different potential categories or the same potential category, but not both. Introducing the idea of category membership under such circumstances would have required 
instructional elaborations that would have excessively complicated the parsing task.

6. Medin et al. (1978), using a paired-associate procedure somewhat different from the one used in Experiment 1, have reported a case in which learning to identify individual category members was inconsistent with the abstraction of shared attributes. The use of a wide variety of training techniques and stimulus materials would be required to fully map the relationship between the abstraction of shared and item-specific attributes.

(Manuscript received May 27, 1986; revision accepted for publication June 5,1987 .) 